\title{
Jaugeage des rivières par ADcp : pour une culture commune
}

\author{
ADcp river discharge measurement : sharing experience \\ JÉRÔME LE COZ \\ Cemagref, Unité de Recherche Hydrologie-Hydraulique, 3 bis quai Chauveau CP 220 F-69336 Lyon cedex 09, France
}

GILLES PIERREFEU

CNR Laboratoire Hydraulique et Mesures, 4 rue de Châlon-sur-Saône F-69007 Lyon, France

\author{
JEAN-FRANÇOIS BROCHOT
}

DIREN Rhône-Alpes SHAC, BP 606 F-21016 Dijon, France

\section{GÉRARD SAYSSET}

EDF DTG, 18 avenue Poincaré, BP 423 F-19311 Brive cedex, France

\section{PIERRE MARCHAND}

IRD Martinique, 3 rue de la rose des vents BP 8006, F-97259 Fort-de-France cedex, France

\begin{abstract}
$T$ The aDcp (acoustic Doppler current profiler) is an efficient tool increasingly used for stream gauging when deployment is possible. Most often, aDcp discharge measurements are in good agreement with standard methods. However, the requirements of correct operation are not fully reported yet and practices are far from unified. Consequently operators, hydrometric network administrators, engineers and research scientists from different French organizations have decided to share ideas about aDcp river discharge measurement, beyond technical support from manufacturers. Within this "Doppler Group", the sharing of varied knowledge and experience, of technological watch, of documents and procedures is a first step towards common culture. The group plans to investigate the uncertainty factors in the aDcp method and to define methodological guidelines through user documents and field operation notes.
\end{abstract}

\section{INTRODUCTION}

Les profileurs acoustiques de vitesse par effet Doppler (aDcp, pour «acoustic Doppler current profiler») sont de plus en plus utilisés pour le jaugeage des cours d'eau, notamment en France depuis une dizaine d'années. Ces appareils apparaissent plus sophistiqués et plus complexes à maîtriser que les techniques conventionnelles d'exploration des vitesses (moulinets, courantomètres électromagnétiques, etc.), mais le calcul du débit repose sur des principes similaires.

Le propos de cet article n'est pas d'entrer dans les détails de l'appareil, mais de prendre le point de vue des utilisateurs en exposant les objectifs et premiers travaux du Groupe Doppler. Ce groupe, créé à Lyon début 2005, se veut un lieu d'échange et de réflexion sur l'outil aDcp. Il souhaite aussi représenter une plate-forme de discussion avec les constructeurs et fournisseurs dont il reste indépendant. En pratique, en partageant leur expérience, leur savoir faire et leurs interrogations, les membres du Groupe Doppler se proposent de constituer une culture commune de la mesure de débit par aDcp.

\section{II — LE GROUPE DOPPLER}

\section{II.1 PROFIL DES UTILISATEURS ET DE LEUR MATÉRIEL}

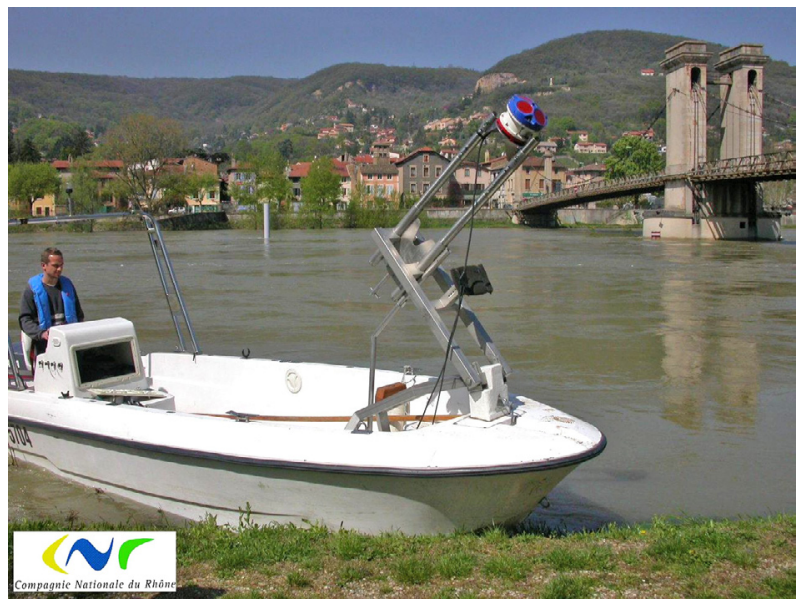

Figure 1 : bras articulé pour le déploiement d'un aDcp solidaire d'un bateau (photo CNR)

Le Groupe Doppler est né du besoin ressenti par de nombreux utilisateurs français d'aDcp de partager leurs expériences pour mieux maîtriser ce nouvel outil, apparaissant souvent comme une boite noire, et pour mieux exprimer leurs besoins méthodologiques. Constitué au tout début de 2005 à l'initiative de la CNR, d'EDF et du Ministère en charge de l'Environnement, le groupe est ouvert à tout utilisateur dési- 
reux d'échanger sur les applications de l'aDcp, en particulier le jaugeage des cours d'eau.

Des gestionnaires, opérateurs et chercheurs représentatifs de différents organismes (cf. Tableau 1) se sont réunis à plusieurs reprises en 2005 pour partager des informations sur les matériels, accessoires, protocoles de jaugeage, difficultés rencontrées, solutions testées, procédures de dépouillement et d'archivage, etc. En relayant les questionnements de la communauté des utilisateurs, le groupe permet de mieux identifier les travaux de recherche et les développements métrologiques nécessaires.

La communication au sein du groupe est facilitée par une liste de diffusion électronique SYMPA (avec archivage des échanges et des documents) hébergée par le serveur informatique du Cemagref à Lyon. Une équipe restreinte, rassemblant les auteurs de cet article, assure la coordination des travaux du groupe. Si les profils des participants sont d'ores et déjà variés, en l'état actuel du groupe, les aDcp concernés

Tableau 1 - profils d'utilisateurs d'aDcp au sein du Groupe Doppler (BB=BroadBand, $W H=$ WorkHorse, 300, 600 ou $1200 \mathrm{kHz}$ )

\begin{tabular}{|c|c|c|c|c|c|}
\hline Organisme & Activités & aDcp (année) & support & Utilisations & $\begin{array}{l}\text { Cours d'eau } \\
\text { jaugés }\end{array}$ \\
\hline $\begin{array}{l}\text { Cemagref } \\
\text { Lyon }\end{array}$ & $\begin{array}{l}\text { recherche et } \\
\text { études }\end{array}$ & WH1200 (2004) & $\begin{array}{c}\text { planche flottante en } \\
\text { mousse }\end{array}$ & $\begin{array}{c}\text { mesures } \\
\text { hydrodynamiques, } \\
\text { sédimentaires et } \\
\text { jaugeages ponctuellement }\end{array}$ & $\begin{array}{c}\text { Rhône, Garonne, } \\
\text { Dordogne }\end{array}$ \\
\hline $\begin{array}{l}\text { CEREGE } \\
\text { Aix-en- } \\
\text { Provence }\end{array}$ & $\begin{array}{l}\text { recherche et } \\
\text { études }\end{array}$ & WH600 & $\begin{array}{l}\text { catamaran non } \\
\text { motorisé }\end{array}$ & $\begin{array}{c}\text { mesures } \\
\text { hydrodynamiques, } \\
\text { sédimentaires et } \\
\text { jaugeages ponctuellement }\end{array}$ & $\begin{array}{c}\text { Bas Rhône et } \\
\text { Camargue }\end{array}$ \\
\hline $\begin{array}{l}\text { CNR } \\
\text { Lyon }\end{array}$ & $\begin{array}{l}\text { gestion de réseau } \\
\text { hydrométrique }\end{array}$ & $\begin{array}{l}\text { BB1200 (1994), } \\
\text { BB600 (1998), } \\
\text { WH600 (2002), } \\
\text { StreamPro (2004) }\end{array}$ & $\begin{array}{c}\text { solidaire bateau / } \\
\text { trimaran non } \\
\text { motorisé }\end{array}$ & $\begin{array}{l}\text { jaugeage, et mesures } \\
\text { hydrodynamiques } \\
\text { ponctuellement }\end{array}$ & Rhône et affluents \\
\hline $\begin{array}{c}\text { DIREN } \\
\text { Centre }\end{array}$ & $\begin{array}{l}\text { gestion de réseau } \\
\text { hydrométrique }\end{array}$ & $\begin{array}{l}2 \text { WH1200 (2000), } \\
2 \text { StreamPro (2005) }\end{array}$ & $\begin{array}{l}\text { planche / solidaire } \\
\text { bateau / catamaran } \\
\text { radiopiloté }\end{array}$ & jaugeage & $\begin{array}{l}\text { Loire, Allier et } \\
\text { affluents }\end{array}$ \\
\hline $\begin{array}{c}\text { DIREN } \\
\text { Rhône-Alpes }\end{array}$ & $\begin{array}{l}\text { gestion de réseau } \\
\text { hydrométrique }\end{array}$ & 3 WH1200, WH600 & $\begin{array}{c}\text { solidaire bateau / } \\
\text { catamaran } \\
\text { radiopiloté }\end{array}$ & jaugeage & $\begin{array}{l}\text { Rhône, Saône, } \\
\text { Doubs et Ain }\end{array}$ \\
\hline $\begin{array}{l}\text { DIREN } \\
\text { Lorraine }\end{array}$ & $\begin{array}{l}\text { gestion de réseau } \\
\text { hydrométrique }\end{array}$ & $\begin{array}{l}\text { WH1200, } \\
\text { StreamPro }\end{array}$ & $\begin{array}{l}\text { solidaire bateau / } \\
\text { catamaran } \\
\text { radiopiloté }\end{array}$ & jaugeage & $\begin{array}{c}\text { Meuse, Meurthe, } \\
\text { Moselle }\end{array}$ \\
\hline $\begin{array}{l}\text { DIREN Nord- } \\
\text { Pas de Calais }\end{array}$ & $\begin{array}{l}\text { gestion de réseau } \\
\text { hydrométrique }\end{array}$ & $\begin{array}{l}\text { WH600 (2005), } \\
\text { WH1200 (2003) }\end{array}$ & $\begin{array}{c}\text { planche / catamaran } \\
\text { radiopiloté }\end{array}$ & jaugeage & $\begin{array}{l}\text { Canaux navigables, } \\
\text { rivières canalisées }\end{array}$ \\
\hline $\begin{array}{l}\text { EDF-DTG } \\
\text { Brive }\end{array}$ & $\begin{array}{l}\text { gestion de réseau } \\
\text { hydrométrique }\end{array}$ & $\begin{array}{l}\text { BB600, WH600, } \\
\text { StreamPro }\end{array}$ & $\begin{array}{c}\text { solidaire bateau / } \\
\text { trimaran non } \\
\text { motorisé }\end{array}$ & jaugeage & $\begin{array}{c}\text { Dordogne, Vienne, } \\
\text { Creuse, Loire, } \\
\text { Allier, Bretagne }\end{array}$ \\
\hline $\begin{array}{l}\text { EDF-DTG } \\
\text { Grenoble }\end{array}$ & $\begin{array}{c}\text { appui et méthode } \\
\text { gestion de réseau } \\
\text { hydrométéo } \\
\text { Alpes }\end{array}$ & $\begin{array}{l}\text { BB600 (1996), } \\
\text { WH600 (2000), } \\
\text { StreamPro (2004), } \\
\text { WH1200 (2004) }\end{array}$ & $\begin{array}{l}\text { catamaran } \\
\text { radiopiloté / } \\
\text { trimaran non } \\
\text { motorisé } \\
\end{array}$ & jaugeage & $\begin{array}{l}\text { Rhône, Isère, Ain } \\
\text { et affluents }\end{array}$ \\
\hline $\begin{array}{l}\text { ENSHMG- } \\
\text { LTHE }\end{array}$ & $\begin{array}{l}\text { recherche et } \\
\text { enseignement }\end{array}$ & - & - & $\begin{array}{l}\text { aucune pour l'instant, } \\
\text { découverte du capteur }\end{array}$ & - \\
\hline IRD & $\begin{array}{l}\text { recherche et } \\
\text { études }\end{array}$ & $\begin{array}{c}\text { BB300, plusieurs } \\
\text { WH (1994 à 1999), } \\
\text { StreamPro (2003) }\end{array}$ & $\begin{array}{l}\text { bateau, parfois } \\
\text { habitable }\end{array}$ & $\begin{array}{c}\text { jaugeage, mesures } \\
\text { sédimentaires }\end{array}$ & $\begin{array}{l}\text { grands fleuves } \\
\text { en Amazonie et } \\
\text { Afrique }\end{array}$ \\
\hline SCHAPI & $\begin{array}{c}\text { prévision et } \\
\text { appui technique }\end{array}$ & - & - & $\begin{array}{l}\text { aucune mais exploitation } \\
\text { de résultats aDcp }\end{array}$ & - \\
\hline
\end{tabular}


sont uniquement issus de la firme californienne Teledyne RDI. Tout utilisateur de profileur acoustique de conception différente - notamment de l'autre principale marque SonTek/ YSI - est en conséquence particulièrement bienvenu.

A terme, le groupe se propose notamment de :

- quantifier, à partir des jaugeages pour lesquels on dispose de données de référence, l'incertitude d'une mesure de débit par aDcp en fonction des conditions de mesure ;

- définir collectivement des bonnes pratiques (mode opératoire, dépouillement et archivage) et des recommandations pratiques (matériels et accessoires) ;

- établir des documents de référence dont un manuel utilisateur commun, une fiche opérateur, etc.

\section{II.2 PRATIQUE DU JAUGEAGE PAR ADCP}

Un premier recensement des activités de jaugeage par aDcp a été analysé, et les principaux résultats synthétiques sont présentés dans le Tableau 2. En dehors du Cemagref qui déploie son instrumentation en fonction des sites supports de ses travaux de recherche, les différents utilisateurs emploient l'aDcp en routine sur les réseaux hydrométriques dont ils ont la charge. Les équipes qui manient l'appareil depuis longtemps (CNR, EDF Brive, DIREN Rhône-Alpes et Centre, IRD Brésil) tendent à privilégier son usage, même si les autres techniques (moulinet, courantomètre, dilution) continuent d'être employées, notamment pour contrôler les mesures par aDcp. Il ne faut pas oublier toutefois que le jaugeage par aDcp reste inadapté voire impossible sur de nombreuses sections (surface trop agitée par exemple) et pour des crues violentes (corps flottants, mise en danger des opérateurs notamment).

Les modes de déploiement au sein du groupe sont divers et adaptés aux sections et aux contraintes locales. Ainsi l'aDcp peut aussi bien traverser le cours d'eau fixé à une embarcation (cf. Figure 1) ou sur un support flottant - planche, catamaran ou trimaran - parfois tracté, parfois motorisé et radiocommandé. Quel que soit le support et la façon de déplacer l'appareil - avec un bateau, depuis un pont, depuis les berges, etc. - la mise à l'eau du matériel est toujours un problème technique à prendre en considération et nécessite parfois des installations adaptées (potence, rampe, etc. cf. Figure 2). Pendant la mesure, les manœuvres doivent être menées le plus souplement et lentement possible, après une période de mise en température.

La gamme de sections jaugées est très étendue, depuis des largeurs de $10 \mathrm{~m}$ et des profondeurs de quelques dizaines de centimètres à des largeurs de plusieurs kilomètres et des profondeurs de l'ordre de $50 \mathrm{~m}$ (cf. Figure 3). Des vitesses moyennes extrêmement faibles (quelques $\mathrm{cm} / \mathrm{s}$ ) ont $\mathrm{pu}$ être mesurées de façon satisfaisante, la limite supérieure se situant aux alentours de $4 \mathrm{~m} / \mathrm{s}$, essentiellement à cause des contraintes de déploiement et de sécurité. Parmi le matériel utilisé au sein du groupe, en complément des ADCP Teledyne RDI «classiques » (BroadBand 300 ou $600 \mathrm{kHz}$, WorkHorse 600 ou $1200 \mathrm{kHz}$ ), le StreamPro est utilisé dans les situations de faibles tirants d'eau $(<2-3 \mathrm{~m})$. Ainsi un WorkHorse Rio Grande $600 \mathrm{kHz}$ et un Streampro $2400 \mathrm{kHz}$ devraient permettre d'assurer le jaugeage dans la plupart des situations. Un panel d'appareils similaires est également proposé par la marque SonTek. Chacun adapte les modes de mesure Teledyne RDI aux configurations des écoulements.

Le savoir faire, les difficultés rencontrées par chacun et l'ensemble des jaugeages archivés et documentés constituent un précieux retour d'expérience à exploiter pour mieux mâ̂triser la mesure par aDcp.

\section{III — RETOUR D'EXPÉRIENCE SUR LES JAUGEAGES ADCP}

\section{III.1 SAVOIR FAIRE ET EXPÉRIENCE À PARTAGER}

Dans des contextes très différents, les utilisateurs ont fait des observations (cf. Tableau 2) et rencontré des difficultés spécifiques (influence de la marée, eaux de fusion) ou génériques (très faibles vitesses entre 0 et $20 \mathrm{~cm} / \mathrm{s}$ ), et ont parfois été amenés à proposer des solutions. Ainsi la CNR comme l'IRD [Callède et al., 2000] ont identifié l'impact de la mobilité du fond sur la mesure de débit et ont développé des méthodes pour en corriger l'effet.

De même, l'examen collectif de cas aberrants (par exemple une valeur de débit s'écartant fortement de la moyenne d'une série de traversées) ou de dysfonctionnements (par exemple, l'impossibilité de mesurer dans les eaux de la Durance en crue) peut aider à progresser. Dans le cas, heureusement rare, des profils aberrants inexplicables par un facteur extérieur (navigation, écoulement instationnaire, mauvaise configuration de l'appareil, etc.), il n'existe pas de critère objectif discriminant autre que l'écart du débit à la moyenne des débits de la série de traversées. Si l'on dispose d'outils de post-traitement permettant de moyenner et de comparer entre eux les champs de vitesse mesurés à travers la section (cf. par exemple Dinehart et al. [2005]), il peut être intéressant de quantifier l'écart du champ de vitesse obtenu sur une traversée par rapport au champ de vitesse moyen sur l'ensemble des traversées. En pratique, une inspection visuelle des vitesses peut suffire à détecter une mesure singulière.

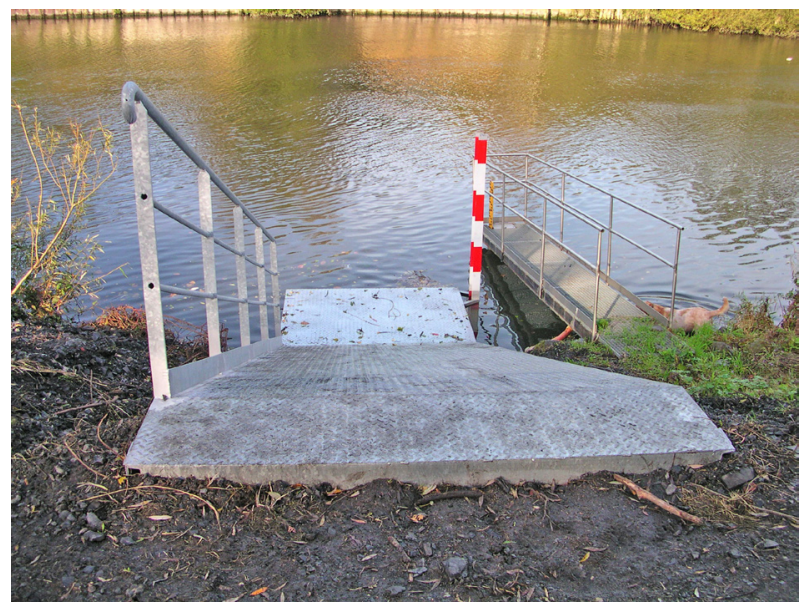

Figure 2 : rampe dédiée à la mise à l'eau de l'aDcp sur support radiocommandé (photo DIREN NPC) 
Tableau 2 - pratique du jaugeage par aDcp au sein du Groupe Doppler

(WH=WorkHorse, $S P=$ StreamPro, BB=BroadBand, $12^{*}:$ mode 12 imposé pour le $S P$ )

\begin{tabular}{|c|c|c|c|c|c|c|c|c|}
\hline $\begin{array}{l}\text { Orga- } \\
\text { nisme }\end{array}$ & $\begin{array}{l}\text { Nombre } \\
\text { de } \\
\text { jaugeages } \\
\text { en } 2004\end{array}$ & $\begin{array}{l}\text { Proportion } \\
\quad \text { de } \\
\text { jaugeages } \\
\text { à l'aDcp }\end{array}$ & $\begin{array}{c}\text { Types } \\
\text { d'aDcp }\end{array}$ & $\begin{array}{c}\text { Largeurs } \\
\text { de section } \\
{[\mathrm{m}]}\end{array}$ & $\begin{array}{c}\text { Profon- } \\
\text { deurs } \\
{[\mathrm{m}]}\end{array}$ & $\begin{array}{c}\text { Vitesses } \\
\text { moyennes } \\
{[\mathrm{m} / \mathrm{s}]}\end{array}$ & $\begin{array}{c}\text { Modes } \\
\text { de mesure } \\
\text { (RDI) }\end{array}$ & Difficultés particulières rencontrées \\
\hline \multirow{2}{*}{$\begin{array}{l}\text { EDF-DTG } \\
\text { Brive }\end{array}$} & \multirow{2}{*}{152} & \multirow{2}{*}{$25 \%$} & WH & 30 à 500 & 0,8 à 5 & 0,05 à 4 & 1581112 & \multirow{2}{*}{$\begin{array}{l}\text { surestimation systématique de } 5-7 \% \text { par } \\
\text { rapport au moulinet ; écart systématique } \\
\text { selon le sens de traversée }\end{array}$} \\
\hline & & & SP & 10 à 250 & 0,3 à 2 & 0,2 à 2 & $12 *$ & \\
\hline $\begin{array}{l}\text { EDF-DTG } \\
\text { Grenoble }\end{array}$ & 250 & $8 \%$ & WH & 25 à 100 & 1 à 4 & 0,5 à 1,5 & 12 & $\begin{array}{c}\text { surface agitée (torrents), sections très } \\
\text { irrégulières ; mesures impossibles pour } \\
\text { certaines natures de particules/bulles } \\
\text { (eaux de fusion, Romanche après } \\
\text { orage...) }\end{array}$ \\
\hline \multirow{2}{*}{$\begin{array}{l}\text { EDF-DTG } \\
\text { Toulouse }\end{array}$} & \multirow{2}{*}{160} & \multirow{2}{*}{$20 \%$} & WH & 25 à 100 & 1 à 9 & 0,05 à 12 & 1581112 & \multirow{2}{*}{$\begin{array}{l}\text { en présence de faible vitesses (Aveyron } \\
\text { par ex.) ; traversées à la corde }\end{array}$} \\
\hline & & & SP & 10 à 12 & - & 0,4 à 0,7 & $12 *$ & \\
\hline \multirow{2}{*}{$\begin{array}{l}\text { CNR } \\
\text { Lyon }\end{array}$} & \multirow{2}{*}{532} & \multirow{2}{*}{$86 \%$} & WH/BB & 5 à 450 & 1 à 20 & 0 à 3 & $1(5)$ & \multirow{2}{*}{$\begin{array}{c}2 \text { à } 5 \text { mesures aberrantes non expliquées } \\
\text { sur plus de } 200 \text { fonds mobiles } \\
\text { en crue ; mesures impossibles dans les } \\
\text { eaux chargées de la Durance en crue }\end{array}$} \\
\hline & & & SP & 2,5 à 200 & 0,3 à 3 & 0 à 2 & $12^{*}$ & \\
\hline $\begin{array}{c}\text { DIREN } \\
\text { Basse- } \\
\text { Normandie }\end{array}$ & - & - & WH & 4 à 35 & 0,5 à 3 & 0,2 à 1,5 & 15 & $\begin{array}{l}\text { mesures parfois faussées à cause de } \\
\text { végétation au fond }\end{array}$ \\
\hline $\begin{array}{c}\text { DIREN } \\
\text { Nord-Pas- } \\
\text { de-Calais }\end{array}$ & - & $10 \%$ & WH & 15 à 70 & $\begin{array}{c}1,5 \text { à } \\
4,5\end{array}$ & 0 à 0,25 & 1512 & $\begin{array}{l}\text { effets transitoires ; perturbations } \\
\text { (navigation, éclusées, marée); } \\
\text { problèmes liés à la végétation; écart } \\
\text { systématique selon le sens de traversée }\end{array}$ \\
\hline $\begin{array}{c}\text { DIREN } \\
\text { Rhône- } \\
\text { Alpes }\end{array}$ & - & $80 \%$ & WH & 10 à 200 & 0,5 à 13 & 0,03 à 4 & 112 & $\begin{array}{l}3 \text { mesures aberrantes (écart }>10 \% \text { ) sur } \\
840\end{array}$ \\
\hline $\begin{array}{l}\text { DIREN } \\
\text { Lorraine }\end{array}$ & - & $20 \%$ & WH & 7 à 125 & 0,8 à 7 & 0,04 à 1,8 & 12 & $\begin{array}{l}\text { écart systématique selon le sens } \\
\text { de traversée (effet supprimé depuis } \\
\text { changement d'embarcation et usage du } \\
\text { mode } 12 \text { en routine) }\end{array}$ \\
\hline $\begin{array}{l}\text { DIREN } \\
\text { Centre }\end{array}$ & 700 & $50 \%$ & WH & 5 à 500 & 0,2 à 10 & 0,05 à 4 & 1 & $\begin{array}{l}\text { fonds mobiles en crue (crue de plus de } \\
3000 \mathrm{~m}^{3} / \mathrm{s} \text { en Loire) } \\
2 \text { mesures sous-estimées d'environ } \\
10 \% \text { comparées à mesures classiques } \\
\text { moulinets en doublon }\end{array}$ \\
\hline $\begin{array}{l}\text { IRD } \\
\text { Brésil }\end{array}$ & - & $100 \%$ & WH/BB & 1 à $10 \mathrm{~km}$ & $\begin{array}{c}\text { jusqu'à } \\
100\end{array}$ & 0,2 à 2 & - & $\begin{array}{l}\text { fonds mobiles en moyennes et } \\
\text { hautes eaux ; marée (avec parfois } \\
\text { des débits négatifs) }\end{array}$ \\
\hline $\begin{array}{c}\text { Cemagref } \\
\text { Lyon }\end{array}$ & - & - & WH & 10 à 300 & 0,8 à 14 & 0,2 à 1 & 181112 & $\begin{array}{l}\text { bathymétrie bruitée en présence de } \\
\text { végétation }\end{array}$ \\
\hline
\end{tabular}

Enfin, la mise en commun du savoir faire technique et de la veille technologique permet de débattre de la pertinence et de la qualité des différents modèles d'aDcp disponibles sur le marché, mais aussi d'accessoires comme le matériel informatique et électronique nécessaire à la communication avec l'aDcp (ordinateur portable, ordinateur de poche, modem radio ou technologie Bluetooth), les supports autonomes (planches, trimarans, catamarans radiopilotés ou non), les systèmes de fixation sur bateau (dans l'axe du bateau ou sur le côté).

\section{III.2 JAUGEAGES EN AUTOCONTRÔLE}

La question qu'amène immédiatement l'emploi d'un appareil aussi nouveau est celle de la précision à attendre sur 
les jaugeages par aDcp, dans les différentes configurations de mesure sur le terrain. Comme l'étalonnage à proprement parler est délicat en laboratoire et pratiquement impossible en conditions naturelles, la question revient en premier lieu à confronter l'aDcp aux méthodes conventionnelles bien documentées (exploration des vitesses par moulinet ou courantomètre électromagnétique, courbe de tarage validée, ouvrage hydraulique dont la loi de débit est connue, etc.). La mise en commun des jaugeages aDcp réalisés en présence de mesures de référence permettra de préciser empiriquement l'incertitude de mesure.

De manière plus ou moins quantifiée, les utilisateurs s'accordent à estimer que l'aDcp est un bon outil de mesure du débit des cours d'eau, c'est-à-dire aussi fiable et précis (sinon plus, dans le cas d'écoulements instationnaires) que les méthodes classiques. Les premières confrontations disponibles auprès des membres du groupe suggèrent que l'écart moyen de mesure de débit à l'aDcp par rapport aux références est typiquement de 5 à $7 \%$. Cette convergence est rassurante, mais elle bute sur au moins deux problèmes de fond : - l'incertitude associée aux mesures conventionnelles est elle-même mal connue, surtout dans les diverses conditions du terrain ;

- les intégrations spatiales et temporelles des vitesses mises en œuvre par la nouvelle méthode ne sont pas les mêmes que pour les autres méthodes. Il convient de s'en souvenir lors de l'élaboration de la grandeur finale « débit» (plus ou moins « instantanée »).

Ce chiffre de 5 à $7 \%$ gagnerait à être confirmé par des études détaillées, en particulier par des analyses d'incertitudes et des tests en laboratoire. C'est un des points importants que le groupe se propose d'aborder.

\section{IV — VERS UNE MÉTHODOLOGIE PARTAGÉE}

\section{IV.1 DOCUMENTS UTILISATEUR}

Les échanges au sein du Groupe Doppler ont permis de réunir et de comparer l'ensemble des documents en fran-

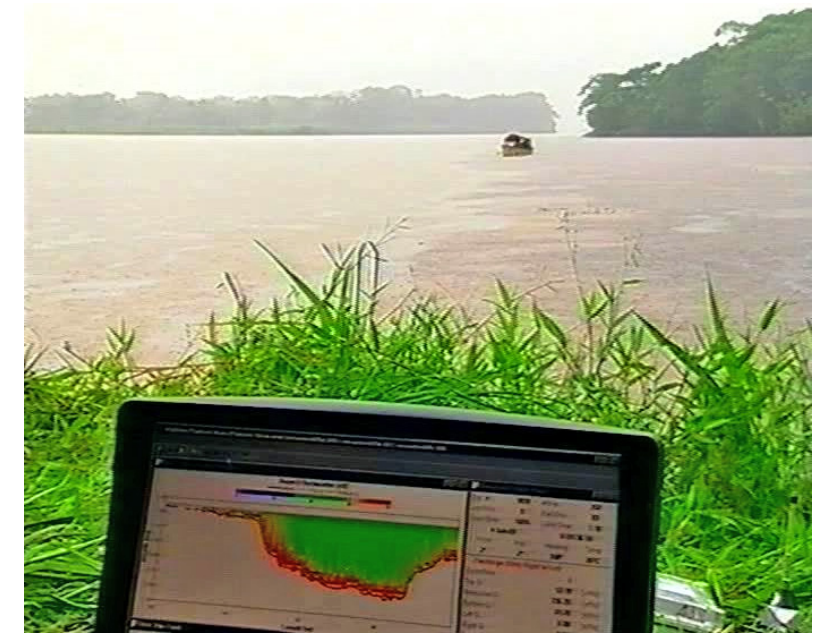

Figure 3 : jaugeage par aDcp en Amazonie (photo IRD)

çais disponibles auprès du Cemagref, de la CNR, de plusieurs DIREN, d'EDF-DTG, et du fournisseur Teledyne RDI France (cf. Tableau 3). Ils ont été confrontés aux publications de l'USGS (United States Geological Survey), l'agence fédérale américaine en charge notamment du suivi des cours d'eau, qui mène depuis de nombreuses années une réflexion indépendante sur le jaugeage par aDcp.

Le Tableau 3 ne détaille pas la diversité des documents produits ou utilisés, comprenant des notes internes, des manuels [Simpson, 2001], des pense-bête (fiche plastifiée EDF par exemple), des traductions de notices, des synthèses bibliographiques et des articles. Le but du Groupe Doppler est ici de croiser toute l'information déjà disponible et utilisée par les membres dans l'optique d'établir un référentiel commun en français. Les aspects les plus importants du point de vue de l'opérateur (en gras Tableau 3) touchent au déploiement du matériel, au déroulement des mesures et à la mise en œuvre du logiciel. Concrètement, il est utile de regrouper si possible dans un seul manuel utilisateur l'en-

Tableau 3 : croisement des documents utilisateur

\begin{tabular}{|c|c|c|c|c|c|c|}
\hline Contenu des documents utlisateur & Cemagref & CNR & DIREN & EDF & USGS & $\begin{array}{c}\text { RDI } \\
\text { France }\end{array}$ \\
\hline Principes de la vélocimétrie acoustique par effet Doppler & $\mathrm{X}$ & - & - & - & $\mathrm{X}$ & $\mathrm{X}$ \\
\hline Principes de la mesure de débit par aDcp & $\mathrm{X}$ & $\mathrm{X}$ & - & - & $\mathrm{X}$ & $\mathrm{X}$ \\
\hline Paramètres de configuration et modes de mesure & - & $\mathrm{X}$ & - & $\mathrm{X}$ & $\mathrm{X}$ & $\mathrm{X}$ \\
\hline Matériel aDcp et accessoires, déploiement & $\mathrm{X}$ & $\mathrm{X}$ & $\mathrm{X}$ & - & $\mathrm{X}$ & $\mathrm{X}$ \\
\hline Protocole de jaugeage / Fiche opérateur & $\mathrm{X}$ & $\mathrm{X}$ & $\mathrm{X}$ & $\mathrm{X}$ & $\mathrm{X}$ & - \\
\hline $\begin{array}{l}\text { Utilisation du logiciel : configuration / acquisition / } \\
\text { dépouillement }\end{array}$ & $\mathrm{X}$ & $\mathrm{X}$ & - & $\mathrm{X}$ & $\mathrm{X}$ & $\mathrm{X}$ \\
\hline $\begin{array}{l}\text { Dépouillement des données, estimation du débit, corrections, } \\
\text { archivage }\end{array}$ & - & $\mathrm{X}$ & - & $\mathrm{X}$ & $\mathrm{X}$ & - \\
\hline Erreurs, problèmes techniques, solutions & - & $\mathrm{X}$ & - & - & $\mathrm{X}$ & - \\
\hline Incertitudes sur le débit, méthodes correctives & - & $\mathrm{X}$ & - & - & $\mathrm{X}$ & - \\
\hline
\end{tabular}


semble des entrées mentionnées, les trois volets en gras pouvant en outre faire l'objet de modèles de fiches synthétiques (recto-verso) utilisables sur le terrain.

\section{IV.2 FICHE OPÉRATEUR ET MODE OPÉRATOIRE}

Les logiciels d'acquisition et de dépouillement de mesures aDcp permettent de renseigner et de stocker une grande quantité d'informations sur le jaugeage au sein des fichiers de données brutes. Ils permettent également d'éditer des comptes rendus synthétiques à partir de ces fichiers bruts. En outre, le logiciel WinRiver1.06 (Teledyne RDI) propose un assistant ( $Q$ Measurements Wizard) pour documenter chaque fichier, à la manière d'un compte rendu d'intervention, mais pour chaque mesure et non pour l'ensemble de la campagne.

Nous estimons que l'usage systématique de fiches opérateurs à remplir sur le terrain offre des avantages indéniables. Une telle fiche guide et structure le déroulement de la mesure, sans risque d'omission ou de désordre : à la fois mémento et tableau de bord, elle donne une vue d'ensemble de la campagne de jaugeage, permettant des ajustements en temps réel. En outre, des remarques de tous ordres peuvent y être consignées librement et rapidement. La fiche opérateur constitue toujours, indépendamment des aléas de l'informatique, un archivage de référence des conditions d'acquisition de la donnée.

Les fiches de terrain développées indépendamment par le Cemagref, la CNR (cf. Figure 4), la DIREN Nord-Pas-deCalais, EDF et l'USGS ont été comparées. Les entrées répertoriées dans le Tableau 4, non exhaustives pour chaque fiche, s'organisent en cinq classes correspondant au déroulement classique d'un jaugeage aDcp :

- Des informations générales servent à référencer la campagne de mesure et à identifier le matériel et les opérateurs. Il est important de bien préciser le lieu exact de la section jaugée qui a été retenue pour ses qualités d'écoulement. En effet contrairement à l'ancienne méthode de jaugeage à la traille, le choix de la section peut différer d'une campagne de jaugeage à une autre. Revenir toujours sur la même section permet toutefois de suivre l'évolution morphologique du lit ;

- Les conditions de mesure à renseigner sont assez disparates, à part le suivi de la cote à l'échelle, pour contrôler la stationnarité de l'écoulement. Les facteurs extérieurs font souvent l'objet de remarques écrites, à l'appréciation des opérateurs ;

- Seuls les paramètres de configuration les plus significatifs sont mentionnés. L'usage d'un fichier de configuration (extension .cfg) ne dispense pas de mentionner les paramètres jugés les plus importants (cf. EDF, USGS);

- Chaque traversée ou chaque point fixe est ensuite décrit, le plus souvent dans un tableau à double entrée, avec une indication du débit total brut calculé par le logiciel d'acquisition. La mesure d'un éventuel déplacement fictif sur un aller-retour (CNR) est un indicateur utile de la mobilité du fond, en vue de corrections ultérieures ;

- Enfin, quelques éléments de synthèse, éventuellement établis de retour au bureau, permettent de conclure la campagne, avant de passer aux phases de dépouillement. Ces indicateurs, s'ils sont connus sur le terrain, peuvent aider à améliorer la mesure : par exemple, réaliser des traversées

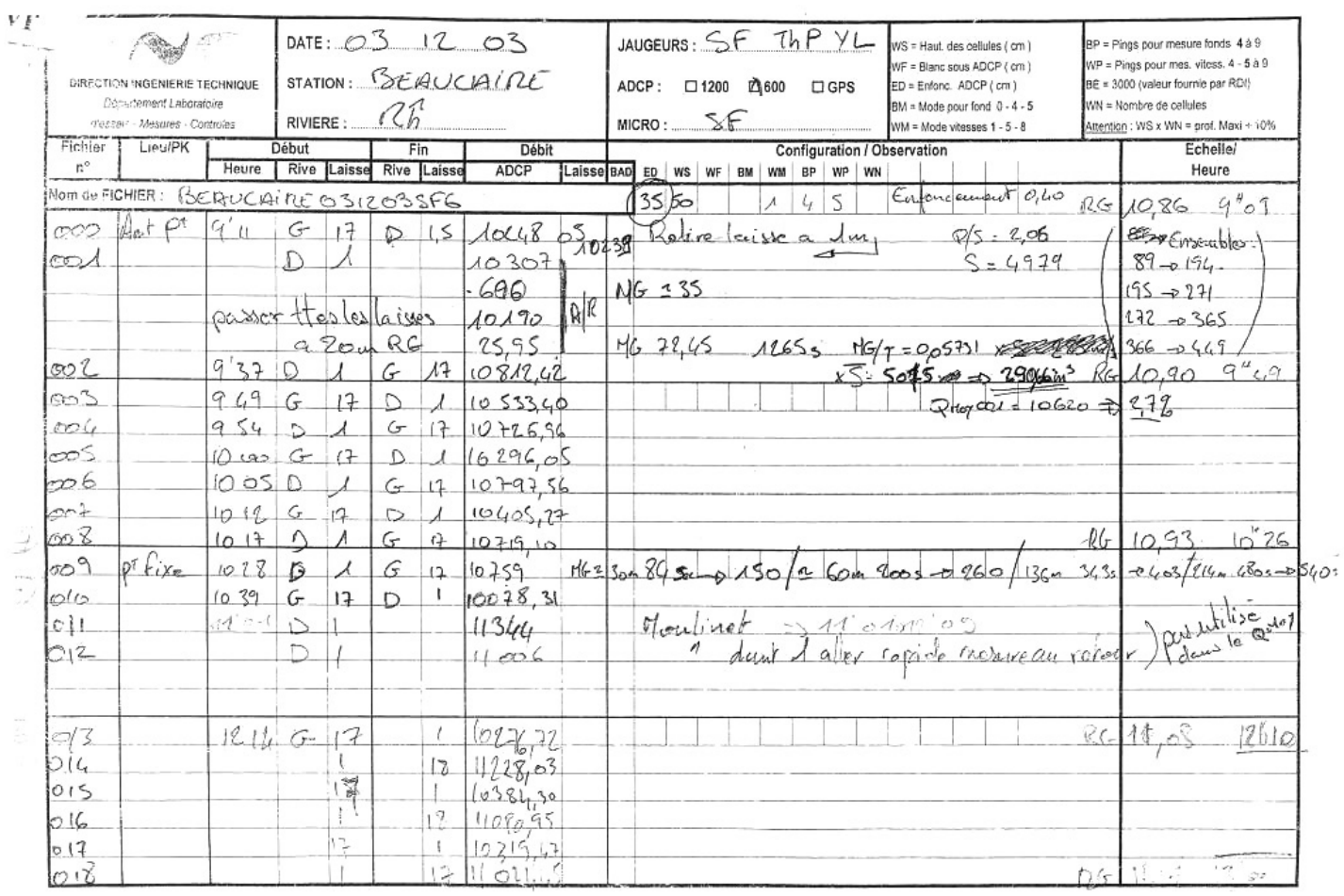

Figure 4 : exemple de fiche terrain (CNR) 
Tableau 4 - croisement des fiches terrain

\begin{tabular}{|c|c|c|c|c|c|c|}
\hline \multicolumn{2}{|c|}{ fiches opérateur (mesures sur le terrain) } & \multirow{2}{*}{$\frac{\text { Cemagref }}{\mathrm{X}}$} & \multirow{2}{*}{$\begin{array}{c}\text { CNR } \\
-\end{array}$} & \multirow{2}{*}{$\begin{array}{c}\begin{array}{c}\text { DIREN } \\
\text { (NPC) }\end{array} \\
\mathrm{X}\end{array}$} & \multirow{2}{*}{$\frac{\text { EDF }}{\mathrm{X}}$} & \multirow{2}{*}{$\begin{array}{r}\text { USGS } \\
\mathrm{X}\end{array}$} \\
\hline général & $\mathrm{n}^{\mathrm{o}}$ fiche & & & & & \\
\hline & date & $\mathrm{X}$ & $\mathrm{X}$ & $\mathrm{X}$ & $\mathrm{X}$ & $\mathrm{X}$ \\
\hline & station & $\mathrm{X}$ & $\mathrm{X}$ & $\mathrm{X}$ & $\mathrm{X}$ & $\mathrm{X}$ \\
\hline & cours d'eau & - & $\mathrm{X}$ & $\mathrm{X}$ & $\mathrm{X}$ & $\mathrm{X}$ \\
\hline & opérateurs & $\mathrm{X}$ & $\mathrm{X}$ & $\mathrm{X}$ & $\mathrm{X}$ & $\mathrm{X}$ \\
\hline & instruments de mesure (dont aDcp) & $\mathrm{X}$ & $\mathrm{X}$ & $\mathrm{X}$ & - & $\mathrm{X}$ \\
\hline & moteur du bateau / type de déploiement & - & - & $\mathrm{X}$ & - & $\mathrm{X}$ \\
\hline & nom générique des fiches & $\mathrm{X}$ & $\mathrm{X}$ & $\mathrm{X}$ & - & $\mathrm{X}$ \\
\hline \multirow[t]{6}{*}{ conditions } & météo & $\mathrm{X}$ & - & - & - & $\mathrm{X}$ \\
\hline & conditions d'écoulement / perturbations & - & - & $\mathrm{X}$ & - & $\mathrm{X}$ \\
\hline & nature du fond / végétation & $\mathrm{X}$ & - & $\mathrm{X}$ & - & - \\
\hline & forme de la section & - & - & - & - & $\mathrm{X}$ \\
\hline & cote à l'échelle (suivi par lecture directe) & $\mathbf{X}$ & $\mathbf{X}$ & $\mathbf{X}$ & $\mathbf{X}$ & $\mathbf{X}$ \\
\hline & température / conductivité de l'eau & $\mathrm{X}$ & - & - & - & $\mathrm{X}$ \\
\hline \multirow[t]{9}{*}{ configuration } & fichier de paramétrage .cfg & - & - & - & $X$ & $X$ \\
\hline & prof. capteur (ED) & - & $\mathrm{X}$ & - & $\mathrm{X}$ & $\mathrm{X}$ \\
\hline & épaisseur cellule (WS) & $\mathrm{X}$ & $\mathrm{X}$ & $\mathrm{X}$ & $\mathrm{X}$ & $\mathrm{X}$ \\
\hline & mode « eau » $(\mathrm{WM})$ & $\mathrm{X}$ & $\mathrm{X}$ & $\mathrm{X}$ & $\mathrm{X}$ & $\mathrm{X}$ \\
\hline & mode « fond $»(B M)$ & $\mathrm{X}$ & $\mathrm{X}$ & - & - & $\mathrm{X}$ \\
\hline & nbre de mesures de fond par ens. (WP) & $\mathrm{X}$ & $\mathrm{X}$ & - & - & $\mathrm{X}$ \\
\hline & nbre de mesures de fond par ens. (BP) & $\mathrm{X}$ & $\mathrm{X}$ & - & - & $\mathrm{X}$ \\
\hline & délai entre deux mesures (TP) & $\mathrm{X}$ & - & - & - & - \\
\hline & méthodes d'interpolation des débits & - & - & - & - & $\mathrm{X}$ \\
\hline \multirow[t]{11}{*}{ traversées } & nom du fichier «transect» & $\mathrm{X}$ & $\mathrm{X}$ & $\mathrm{X}$ & $\mathrm{X}$ & $\mathrm{X}$ \\
\hline & heure départ & - & $\mathrm{X}$ & $\mathrm{X}$ & $\mathrm{X}$ & $\mathrm{X}$ \\
\hline & rive départ & $\mathrm{X}$ & $\mathrm{X}$ & $\mathrm{X}$ & $\mathrm{X}$ & $\mathrm{X}$ \\
\hline & distance / rive & $\mathrm{X}$ & $\mathrm{X}$ & $\mathrm{X}$ & $\mathrm{X}$ & $\mathrm{X}$ \\
\hline & heure arrivée & - & - & - & - & $\mathrm{X}$ \\
\hline & rive arrivée & - & $\mathrm{X}$ & - & $\mathrm{X}$ & $\mathrm{X}$ \\
\hline & distance / rive & - & $\mathrm{X}$ & $\mathrm{X}$ & $\mathrm{X}$ & $\mathrm{X}$ \\
\hline & déplacement fictif aller-retour (MG) & - & $\mathbf{X}$ & - & - & - \\
\hline & débit total brut & $\mathrm{X}$ & $\mathrm{X}$ & $\mathrm{X}$ & $\mathrm{X}$ & $\mathrm{X}$ \\
\hline & détail débits masuré / interpolés & - & - & - & $\mathrm{X}$ & $\mathrm{X}$ \\
\hline & $\%$ débit mesuré & - & - & - & - & $\mathrm{X}$ \\
\hline \multirow[t]{3}{*}{ synthèse } & moyenne des débits successifs & $\mathrm{X}$ & - & $\mathrm{X}$ & $\mathrm{X}$ & $\mathrm{X}$ \\
\hline & dispersion des débits successifs & - & - & $\mathrm{X}$ & - & $\mathrm{X}$ \\
\hline & pourcentage du débit final mesuré / total & - & - & - & - & $\mathrm{X}$ \\
\hline
\end{tabular}


supplémentaires si la dispersion des débits successifs s'avère trop élevée.

L'objectif d'un tel croisement est de faire ressortir la structure et les entrées que devrait proposer une fiche de jaugeage par aDcp «standard», adaptable si besoin aux procédures spécifiques à chaque organisme. La constitution d'un tel prototype de fiche terrain est indissociable de l'élaboration d'un mode opératoire commun, précisant en premier lieu le nombre et le type de traversées à effectuer. C'est un objectif à court terme pour le groupe.

Quelques pistes se dégagent pour l'instant:

- assurer la mise en température du capteur et vérifier le bon fonctionnement du thermomètre de l'aDcp ;

- faire un - voire deux pour confirmer - aller/retour au même repère fixe sans arrêter l'enregistrement permet de quantifier un éventuel déplacement du fond, tout en procédant aux premières mesures de débit;

- réaliser un minimum de 4 voire 6 traversées (autant dans chaque sens si possible) permet de s'assurer de la stabilité des résultats.

Il convient enfin de définir les informations pertinentes qui relèvent des opérations sur le terrain, et celles qui trouveront plutôt place dans des comptes rendus plus détaillés (données élaborées).

\section{IV.3 DÉPOUILLEMENT, CRITIQUE DES DONNÉES, ARCHIVAGE}

Une fois la campagne de mesure achevée, un certain nombre de tâches doivent être effectuées pour exploiter, contrôler et conserver les données acquises. En attendant une analyse plus approfondie à partir des pratiques en cours et des problèmes identifiés, trois points nous paraissent susceptibles d'être précisés :

- Etablir, sur des principes métrologiques objectifs, le débit jaugé lors d'une série de traversées. En particulier, les traversées donnant des valeurs de débit aberrantes selon des critères prédéfinis ne devraient pas être écartées si aucune cause d'erreur n'est identifiée formellement [Lipscomb, 1995]. Il est préférable de moyenner autant de traversées dans chaque sens pour minimiser l'impact d'un éventuel biais directionnel [Oberg et al., 2005]. Une importance particulière doit être accordée au choix de la section et aux conditions de mesure : en premier lieu vérifier la stabilité des écoulements via le suivi de la cote à l'échelle ou sur une station de référence comme les stations à ultrasons ;

- Définir des indicateurs de la qualité d'un jaugeage en attendant qu'une estimation rigoureuse des incertitudes soit réalisable en pratique. Pour une traversée, le rapport du « débit mesuré » correspondant à la partie explorée de la section, au « débit total » après extrapolation des vitesses aux zones inaccessibles à la mesure devrait ainsi apparaître sur le compte rendu de jaugeage. En effet, on peut penser que si ce ratio est trop faible, les incertitudes sur le jaugeage seront fortes. Toutefois les auteurs ont observé de nombreux cas où avec un ratio de seulement $30 \%$ la valeur de débit fournie par l'aDcp s'avère cohérente. Quand la surface de section non explorée est importante, la pertinence des lois d'extrapolation des vitesses devient déterminante pour la qualité du jaugeage ;

- Archiver les données brutes et élaborées, en assurant leur intégrité (les fichiers bruts sont très volumineux), leur accessibilité (classement rigoureux) et leur pérennité (en particulier les formats de fichiers liés à des logiciels spécifiques peuvent rapidement devenir caducs). Les procédures d'archivage sont bien sûr propres à chaque organisme mais elles peuvent respecter des principes établis ensemble.

Ici aussi, l'établissement d'une méthodologie de référence et la diffusion d'outils de dépouillement et d'archivage amélioreraient l'exploitation des données aDcp. Par exemple, la DIREN NPC a développé et utilise un utilitaire informatique pour stocker automatiquement les données brutes de chaque campagne de mesure selon un classement standardisé.

\section{PERSPECTIVES}

Les travaux du Groupe Doppler en 2005 ont permis de dresser un premier état des lieux de la pratique de l'aDcp au sein des différentes équipes et des principales pistes à investiguer. La mise en réseau des expériences se poursuivra à travers les réunions régulières et la liste de diffusion électronique. Une analyse plus approfondie des jaugeages aDcp passés - en particulier ceux que l'on peut confronter à une autre méthode de mesure - devrait permettre au groupe de proposer des critères objectifs de rejet de traversées aberrantes ainsi que des estimations empiriques des gammes d'incertitude. Enfin, le groupe va synthétiser ses informations et son expérience en vue de la réalisation d'un manuel utilisateur (à la fois pédagogique et méthodologique) et de modèles de fiches terrain (matériel, logiciel et mode opératoire) adaptables aux contraintes et aux procédures de chacun, notamment en termes de contrôle et de suivi du matériel.

\section{V — REMERCIEMENTS}

Nous sommes reconnaissants à la DIREN Rhône-Alpes pour avoir accueilli les réunions du Groupe Doppler au sein de ses locaux lyonnais. Merci aux utilisateurs d'aDcp participant aux travaux du groupe et à bien d'autres encore ayant réalisé des mesures par aDcp et nous ayant transmis des informations.

\section{REFERENCES}

[1] Callède J., Kosuth P., Guyot J.L., \& Santos Guimaraes V. (2000). - Discharge determination by ADCP : a moving bottom error correction method and its application on the river Amazon at Obidos. Hydrol. Sci. J., 45 (6) : 911-924.

[2] Dinehart R.L., \& Burau J.R. (2005). - Averaged indicators of secondary flow in repeated acoustic Doppler current profiler crossings of bends. Wat. Resour. Res., 41 (9) : 1-18.

[3] Lipscomb S.W. (1995). - Quality-assurance plan for discharge measurements using broadband acoustic Doppler current profilers, USGS open-file report 95-701, 5 p. 